\title{
Paper
}

\section{Prediction Model of Light-induced Melatonin Suppression}

\author{
Yoshika TAKAHASHI, Tetsuo KATSUURA, Yoshihiro SHIMOMURA and Koichi IWANAGA
}

Chiba University

Paper originally published in Japanese in IEIJ, Vol.94 No.2, 2010

A part of this article was presented by Yoshika Takahashi at Color Forum Japan 2007 and the 42nd Annual Conference of IEIJ in 2009.

\begin{abstract}
The prediction method of melatonin suppression values was based on previous studies related to melatonin suppression and pupil constriction. Estimated values that considered pupil constriction were larger than the actual suppression values. We focused on the pupil constriction and its correction factor to interpret the action spectrum for the properties of the melatonin suppression model. When the correction factor was used to modify the model, actual suppression values were almost completely predictable. These factors suggest that it might be possible to explain the indescribable results.
\end{abstract}

KEYWORDS: circadian, melatonin, action spectrum, pupil, photoreceptor, suprachiasmatic nucleus

\section{Introduction}

Light not only provides humans with the perception of information such as color, shape, and brightness, but also affects various bodily functions. Color, shape, and brightness are perceived after light signals received by the retina pass through the lateral geniculate nucleus (LGN) and then reach the cerebral cortex. The effects on bodily functions are believed to be related to the passage of light signals to the pineal gland ${ }^{1}$. The light signals received by the retina enter the suprachiasmatic nucleus (SCN) and then pass through the paraventricular nucleus (PVN), medial forebrain bundle (MFB), and reticular formation (RF) to the pineal gland. The pathway related to color, shape, and brightness is referred to as the visual pathway, while the pathway related to the effects on bodily functions is referred to as the nonimage forming pathway.

In the past, investigations of the effects of light on bodily functions have covered the effects on the central nervous system, autonomic nervous system, and endocrine system ${ }^{2)-19)}$. Effects on brain waves (EEG) and event-related potential (ERP) have been reported as marks of the central nervous system ${ }^{2)-13)}$, effects on blood pressure, pulse, skin temperature, electrodermal activity (EDA), and heart rate variability (HRV) have been reported as marks of the autonomic nervous system ${ }^{34) 14)-16)}$, and the effects on melatonin, cortisol, and thyroid stimulation hormone (TSH) have been reported as marks of the endocrine system ${ }^{17)-19)}$. When the effects of light on the central and autonomic nervous systems are categorized by color, experimental results can generally be divided into two groups. One group indicates that the color described as red (a color with a large longwavelength component) has an arousal effect and the color described as blue (a color with a large short-wavelength component) has a calming effect(2)-4)6)8)10)-12)14)16). Conversely, the other group shows that the color described as red has a calming effect and the color described as blue has an arousal effect5)-9)13)15). The fact that there are multiple reports showing that the effects of light on bodily functions can have the exact opposite results makes it conceivable that these are not the results of artifacts but are the results of causes. When the research produces the similar results, the spectral distribution features of the light source used are similar. In experiments that produce results showing that red and blue have arousal and calming effects, respectively2)-4)6(8)10)-12)14)16), color fluorescent lamps and colored light that was filtered from incandescent lamps were used. In experiments that produce results showing that red and blue have calming and arousal effects, respectively5)-9)13)15), fluorescent lamps that have chromaticity in the Planckian locus range that can be expressed by correlated color temperature and monochromatic light that was filtered by a narrowband transmission interference filter from an incandescent lamp were used. These results suggest that the results of the effects of light on bodily functions cannot be simply expressed by a color name. There is a complicated mechanism that changes these effects according to the spectral distribution shape.

Many reports on the results of the effects of light on the endocrine system have covered the effects on melatonin that is secreted mainly at night by the pineal gland. The secretion of melatonin is rapidly suppressed 
by high-intensity light at night17). In experiments that used color fluorescent lamps ${ }^{20)}$, green and blue lights greatly suppressed melatonin secretion, while red light had almost no suppression effect. For all experiments that used fluorescent lamps that have chromaticity in the Planckian locus range ${ }^{21)}$ or monochromatic light ${ }^{2223)}$, it was reported that light with a large short-wavelength component, which is described as blue, strongly suppressed melatonin secretion. The action spectrum for melatonin suppression from light has been studied, and it has been reported that light in the range of 446 to 477 $\mathrm{nm}$ has the largest effect ${ }^{22}$. Furthermore, intrinsically photosensitive retinal ganglion cells (ipRGC) that contain the photopigment melanopsin have been discovered in recent years. These cells are photoreceptors but are distinct from rods and cones ${ }^{24}$. Their neural output projects to some layers of the SCN and LGN (IGL, VLG) and the olivary pretectal nucleus $(\mathrm{OPN})^{25}$. The fact that the first 3 locations are related to the circadian rhythms and that the OPN is related to pupil reflex suggests that the ipRGC transmit light signals via non-image forming pathways. Experiments using mice that lost melanopsin or rod and cone function ${ }^{26)}$ showed that while mice that lost melanopsin function only or rod and cone function only showed melatonin suppression and pupil reflex, mice that lost the function of all three photoreceptors lost all reaction to light. This suggests that ipRGC has an important function along with rods and cones in melatonin suppression and pupil reflex reactions. Experiments that compared melatonin suppression by narrowband monochromatic light and broadband light, which includes wavelengths of multiple bands, suggest that the melatonin suppression effects of broadband light are lower than predicted by action spectrum ${ }^{2223)}$ models ${ }^{27)}$ of melatonin suppres$\operatorname{sion}^{28) 29}$. While it is possible to roughly estimate the effect of melatonin suppression by the color of the light, the additivity of the action spectrum has been called into doubt in some reports ${ }^{2829)}$, which suggests that there is some not-yet-understood complex mechanism at work.

While the effects of light on bodily functions are complex, it is important to quantify the results and create design criteria for lighting environments that take into account the impact of these results. Consequently, this report aims to use findings related to one of the effects of light on bodily functions, the effect on melatonin secretion, to create a model to estimate the melatonin suppression value caused by exposure to light at night. Within the marks that have circadian rhythms, such as sleep and waking, rectal temperature, and cortisol, the secretion rhythm of melatonin is said to be the least effected by the actions other than those of the biological clock in SCN. Because there are basic findings regard- ing multiple factors of the effects of light on melatonin secretion, such as action spectrum ${ }^{22) 23}$, the dose $\mathrm{re}^{-}$ sponse relation with light intensity ${ }^{2223)}$, duration of $\mathrm{ex}^{-}$ posure to light ${ }^{30) 31 \text {, }}$, pupil reflex ${ }^{32}$, and age ${ }^{33 \text { ), they are }}$ considered appropriate marks when modeling the influence of light on living organisms. In many reports that covered the influence of light on melatonin secretion, mydriatics are used and pupil diameter does not change in the experiment so the conditions differ from general environments which includes pupil reflex. Therefore, this report presents mathematical models that predict the melatonin suppression value under conditions in which pupil reflex changes the quantity of light reaching the retina.

\section{Mathematical models for melatonin suppression}

Firstly, a mathematical model was created for conditions with no pupil reflex. Next, a model was created for conditions with pupil reflex. The mathematical model for conditions with no pupil reflex was created from knowledge related to action spectrum and doseresponse curves based on experiments that clarified the action spectrum of melatonin suppression. It has been reported that conditions with pupil reflex have a lower melatonin suppression value compared to conditions with no pupil reflex ${ }^{32}$. It is believed that the pupil reflex attenuates the amount of light that reaches the retina and causes this difference. The formula for conditions with no pupil reflex was created by transforming a formula that focuses on the quantity of light reaching the pupil.

\subsection{Action spectrum for melatonin suppression}

The action spectrum for melatonin suppression has been carefully studied by Brainard et al. ${ }^{22}$ and Thapan et al. ${ }^{23)}$, who reported similar results. To predict the melatonin suppression value for conditions with pupil reflex later in this report, it was decided to use the experimental results of Brainard et al. ${ }^{22)}$, which list pupil diameter at dilation.

It has been reported that the melatonin suppression from light exposure differs by the time of day of the light exposure ${ }^{34)}$. Brainard et al. ${ }^{22}$ ) used the method of Gaddy et al. ${ }^{32}$ to cancel the influence of the time of day of the light exposure to calculate the melatonin suppression value. Formula 1 was used to calculate the melatonin suppression value with and without light exposure. Next, the value without exposure was subtracted from the value with exposure. After control-adjusting the value, the melatonin suppression value was acquired.

$$
100 \times \frac{\text { melatonin }(3: 30)-\text { melatonin }(2: 00)}{\text { melatonin }(2: 00)}
$$


melatonin (3:30) : Melatonin concentration at 3:30 melatonin (2:00) : Melatonin concentration at 2:00

The dose-response curve expressing the relation of the light intensity for each monochromatic light and the melatonin suppression value after control-adjustment is expressed in Formula 2 by a logistic function with the 4 types of parameters indicated ${ }^{22}$.

$$
M S \%=\frac{A_{1}-A_{2}}{1+\left(X / X_{50}\right)^{p}}+A_{2}
$$

$$
\begin{array}{cl}
M S \% & : \text { Melatonin suppression value } \\
X & : \text { Light intensity } \\
A_{1} & : \text { Minimum melatonin suppression value } \\
A_{2} & : \text { Maximum melatonin suppression value } \\
X_{50}: & \text { The light intensity that produces a mela- } \\
& \text { tonin suppression value halfway between } \\
& \text { the minimum and maximum } \\
p \quad: \text { Slope of curve }
\end{array}
$$

The action spectrum was calculated from the logistic function of Formula 2 created for each monochromatic light. The three parameters other than $X_{50}$ were made common, and then $X_{50}$ for each monochromatic light was calculated. The values in the report by Glickman et al. ${ }^{35)}$ were used for $X_{50}$. The action spectrum was calculated by reciprocating and normalizing $X_{50}$. The unit 'photon' is frequently used with the original $X_{50}$ used to calculate the action spectrum, which is different from the general energy unit used in engineering. Therefore, the unit was converted using Formula 3 below.

$$
\begin{aligned}
& Q=\frac{h \cdot c}{\lambda} \\
& Q \quad \text { : Energy of one photon (J) } \\
& h \text { : Planck's constant }\left(\mathrm{J} \mathrm{s}_{\mathrm{s}}\right) \\
& c \text { : Light-speed in vacuum }(\mathrm{m} / \mathrm{s}) \\
& \lambda \text { : Wavelength of light }(\mathrm{m})
\end{aligned}
$$

Table 1 shows $X_{50}$ in units of photons (photon flux density) and energy (irradiance) and the action spectrum in units of energy. Furthermore, Brainard et al. ${ }^{22)}$ identified the importance of investigating the action spectrum of short wavelengths of less than $440 \mathrm{~nm}$ and so an experiment using monochromatic light with the

Table 1 Radiometric quantity of half saturation constant $\left(X_{50}\right)$ in eight different wavelengths and action spectrum based on energy ${ }^{22) 35)}$

\begin{tabular}{cccccccccc}
\hline & $440 \mathrm{~nm}$ & $460 \mathrm{~nm}$ & $480 \mathrm{~nm}$ & $505 \mathrm{~nm}$ & $530 \mathrm{~nm}$ & $555 \mathrm{~nm}$ & $575 \mathrm{~nm}$ & $600 \mathrm{~nm}$ \\
\hline \multirow{2}{*}{$X_{50}$} & $\begin{array}{c}\text { Photon flux density } \\
\left(\times 10^{12} \text { photons } / \mathrm{cm}^{2} / \mathrm{sec}\right)\end{array}$ & 5.35 & 5.59 & 8.28 & 8.33 & 18.01 & 77.5 & 135 & 333 \\
\cline { 2 - 10 } & Irradiance $\left(\mu \mathrm{W} / \mathrm{cm}^{2}\right)$ & 2.42 & 2.41 & 3.43 & 3.28 & 6.75 & 27.7 & 46.6 & 110 \\
\hline Relative energy sensitivity & 0.999 & 1.00 & 0.704 & 0.737 & 0.358 & 0.0870 & 0.0518 & 0.0219 \\
\hline
\end{tabular}

wavelength of $420 \mathrm{~nm}$ was added. However, it was decided that the action spectrum of $420 \mathrm{~nm}$ should not be used because there was only one light intensity condition and the precision was considered poor.

\subsection{Approximation formula for the action spectrum of} melatonin suppression

It is been reported that the action spectrum of melatonin suppression has a peak wavelength of around 460 $\mathrm{nm}^{2223)}$. This is different than both the standard relative luminous efficacy function for photopic vision $V(\lambda)$ and the standard relative luminous efficacy for scotopic vision $V^{\prime}(\lambda)$. Since both the rod and cone system and ipRGC take part in this mechanism, the action spectrum was approximated using a quadratic polynomial (Formula 4) instead of the spectral absorption characteristics of individual photoreceptors ${ }^{2223)}$ or a synthesis of multiple photoreceptors of the rod and cone system ${ }^{27}$.

$$
\begin{aligned}
& M(\lambda) L=-1.164 \times 10^{2} \cdot t^{2}+4.029 \times 10^{-2} \cdot t \\
& M(\lambda)=10^{M(\lambda) L} \\
& t=\log \left(\lambda / \lambda_{\max }\right) \\
& \lambda_{\max }=453 \times 10^{-9}
\end{aligned}
$$

$M(\lambda) L$ : Approximation formula when expressed as a common logarithm

$M(\lambda)$ : Approximation formula for action spectrum of melatonin suppression based on the $\mathrm{ex}^{-}$ perimental results of Brainard et al. ${ }^{22}$ )

$t$ : Wavelength function

$\lambda$ : Wavelength $(\mathrm{m})$

$\lambda_{\max }:$ Peak wavelength of approximation formula (m)

Figure 1 shows the results for the action spectrum for melatonin suppression ${ }^{22}$, approximation using the tem-

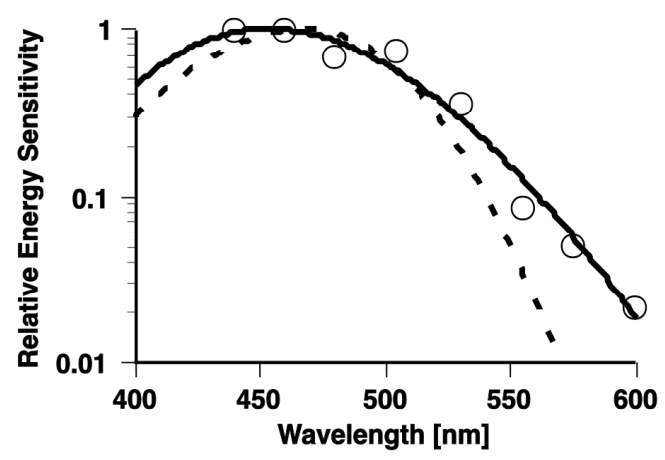

Figure 1 The open circle represents the action spectrum for melatonin suppression ${ }^{22)}$. The broken curve represents template for maximal spectral absorbance of $464 \mathrm{~nm}^{22}$. The bold curve represents quadratic polynomial. 
plate for the spectral absorbance characteristics of a visual pigment with a peak wavelength of $464 \mathrm{~nm}$ used by Partridge et al. $\left(\mathrm{R}^{2}=0.91\right)^{36}$, and approximation using the quadratic polynomial presented in this report $\left(\mathrm{R}^{2}=0.98\right)$. This figure shows that the template with a peak wavelength at $464 \mathrm{~nm}$ does not match well with the experimental results and the approximation curve when the wavelength is longer than $550 \mathrm{~nm}$. In contrast, the quadratic polynomial presented in this report matches well with the experimental results of Brainard et al. ${ }^{22)}$ over the entire range of 440 to $600 \mathrm{~nm}$ used in the experiment.

\subsection{Mathematical model without pupil reflex}

A single dose-response curve that is not dependent on the wavelength of light can be created by clarifying the action spectrum from a dose-response curve acquired using monochromatic light, and then expressing it as a function of the amount corresponding to the illuminance using this action spectrum. In order to create a single dose-response curve based on the experimental results of Brainard et al. ${ }^{22}$ ) that is not dependent on the wavelength of light, it is necessary to express $X_{50}$ of Formula 2 as an amount corresponding to the illuminance using the action spectrum.

In order to calculate $X_{50}$ as an amount corresponding to the illuminance using the action spectrum, we assume the experimental conditions used by Brainard et al. ${ }^{22)}$ and calculated the melatonin suppression value at that time (the experimental results). The experimental conditions used by Brainard et al. ${ }^{22}$ ) were assumed from the figure in the report. The photon flux density ranges set for each monochromatic light were as follows: 440 $\mathrm{nm}: 1$ to $316 \times 10^{12}$ photons $/ \mathrm{cm}^{2} / \mathrm{sec}, 460,480,505,530$ $\mathrm{nm}: 3.16$ to $100 \times 10^{12}$ photons $/ \mathrm{cm}^{2} / \mathrm{sec}, 555,575 \mathrm{~nm}: 10$ to $316 \times 10^{12}$ photons $/ \mathrm{cm}^{2} / \mathrm{sec}, 600 \mathrm{~nm}: 31.6$ to $316 \times 10^{12}$ photons $/ \mathrm{cm}^{2} / \mathrm{sec}$. The light intensity interval was 0.25 log step and the number of intensity conditions were as follows: $440 \mathrm{~nm}: 11 ; 460,480,505,530,555,575 \mathrm{~nm}: 7$; $600 \mathrm{~nm}: 5$. The experimental results for Brainard et al. ${ }^{22)}$ use the $X_{50}$ of each monochromatic light listed in the report of Glickman et al.35) (Table 1) and the melatonin suppression value was calculated for the monochromatic lights and light intensities assumed above. The spectral distribution of monochromatic light was created using a gauss function. The full width at half maximum (FWHM) was $10 \mathrm{~nm}$.

Next, the amount corresponding to the illuminance using $M(\lambda)$ was calculated for the assumed experimental conditions. Formula 5 shows the calculation formula.

$$
\begin{aligned}
& E_{m}=K_{m} \int_{380}^{780} P_{t}(\lambda) \cdot M(\lambda) d \lambda \\
& K_{m}=5450
\end{aligned}
$$

$E_{m}$ : The amount corresponding to the illuminance using $M(\lambda)$

$K_{m}$ : Coefficient to set the value of $555 \mathrm{~nm}$ for $M(\lambda)$ to 683

$P_{t}(\lambda)$ : Spectral power distribution of light source $\left(\mathrm{W} / \mathrm{m}^{2}\right)$

$M(\lambda)$ : Approximation formula for the action spectrum of melatonin suppression based on the experimental results of Brainard et al. ${ }^{22)}$

Finally, the results were plotted with the amount corresponding to the illuminance calculated above on the horizontal axis and the melatonin suppression value under the experimental conditions of Brainard et al. ${ }^{22)}$ on the vertical axis. The curve that represents this plot was calculated using the logistic function of Formula 2. Next, $X_{50}$ was calculated with $A_{1}, A_{2}$, and $p$ at the same values used by Brainard et al. ${ }^{22)}$. The result for $X_{50}$ was 131. Formula 6 shows the relational expression for the acquired melatonin suppression value.

$$
\text { MS\% } \% \text { dilated }=\frac{0-66.9}{1+\left(E_{m} / 131\right)^{1.27}}+66.9
$$

$M S \%$ dilated: Melatonin suppression value when pupils are dilated

$E_{m}$ : The amount corresponding to the illuminance using $M(\lambda)$.

\subsection{Prediction formula for pupil diameter}

Stanley et al. ${ }^{37}$ ) reported on the relation of pupil diameter and the brightness of the surrounding environment. Pupil diameter $[\mathrm{mm}]$ is expressed by Formula 7 as a function of the corneal flux density $F\left[\mathrm{~cd} / \mathrm{m}^{2} \cdot \mathrm{deg}^{2}\right]$, which is expressed by the luminance $\left[\mathrm{cd} / \mathrm{m}^{2}\right]$ and the squaring of the visual angle [deg].

$$
\begin{aligned}
& D=7.75-5.75\left\{\frac{(F / 846)^{0.41}}{(F / 846)^{0.41}+2}\right\} \\
& D \quad \text { : Pupil diameter (mm) } \\
& F \quad \text { : Corneal flux density }\left(\mathrm{cd} / \mathrm{m}^{2} \cdot \mathrm{deg}^{2}\right)
\end{aligned}
$$

As with the melatonin suppression value, pupil diameter can be conveniently calculated from illuminance and the spectral distribution of the light source. The light exposure device used by Brainard et al.22) covered the full field of vision of the subjects by using a spherical device. In this device, spectral monochromatic light was produced by a monochromator. In the experiment by Stanley et al. ${ }^{37)}$ that reported on the relation between pupil diameter and the brightness of the surrounding environment, pupil diameter was measured as subjects 
observed circular light stimuli in various visual angles range from 0.4 to $25.4 \mathrm{deg}$. and under various luminance conditions. Although the conditions presenting light stimuli to the subjects were different, it was assumed that it was possible to calculate the pupil diameter under the experimental conditions of Brainard et al. ${ }^{22)}$ using the relational formula of Stanley et al. ${ }^{37)}$ at this time. As with the mathematical model to predict melatonin suppression, pupil diameter was acquired by entering the illuminance and light source spectral distribution information. To do this, it is necessary to know the relation of "corneal flux density $F$ " of Formula 7 and "illuminance and light source spectral distribution".

Illuminance can be expressed as a function of luminance or surface light source area and the distance from the light source ${ }^{38}$. When the surface light source and side light surface are opposed, $\theta=0$ and $\phi=0$. The surface light source is assumed to be circular, the radius of the circle is $r$, and the visual angle of the circular surface light source is $\delta$. The resulting formula is Formula 8. If the surface light source and side light surface are opposing and the surface light source is assumed to be circular, this formula can calculate illuminance $E$ [lx] from luminance $L\left[\mathrm{~cd} / \mathrm{m}^{2}\right]$ and visual angle $\delta$ [deg].

$$
\begin{aligned}
& E=L \cdot \Delta S \cdot \cos \theta \cdot \cos \phi / D^{2} \\
& =L \cdot \Delta S / D^{2} \\
& =L \cdot \pi \cdot r^{2} / D^{2} \\
& =L \cdot \pi \cdot \tan ^{2}(\delta / 2)
\end{aligned}
$$

When $F$ is expressed as $E$, the formula is transformed into Formula 9. When the visual angle conditions $\delta$ used by Stanley et al. ${ }^{37)}(0.4,1.6,7.4,25.4 \mathrm{deg})$ are entered, $\delta$ becomes $4180,4179,4168,4044$, respectively. When a weighted average is calculated in relation to the number of visual angle conditions used by Stanley et al. ${ }^{37}$, the $\delta$ is 4135 .

$$
\begin{aligned}
& F=L \cdot \delta^{2} \\
& =\frac{E}{\pi \cdot \tan ^{2}(\delta / 2)} \cdot \delta^{2} \\
& =4135 \cdot E
\end{aligned}
$$

When Formula 9 is applied to the Formula 7, Formula 10 is generated, which can calculate the pupil diameter $D$ from illuminance $E$.

$$
\begin{gathered}
D=7.75-5.75\left\{\frac{(4135 \cdot E / 846)^{0.41}}{(4135 \cdot E / 846)^{0.41}+2}\right\} \\
D \quad: \text { Pupil diameter }(\mathrm{mm}) \\
E \quad: \text { Illuminance }(\mathrm{lx})
\end{gathered}
$$

It has also been reported that pupil diameter accommodates to the spectral sensitivity of rods ${ }^{39}$. Because Stanley et al. ${ }^{37}$ ) used a light source that had a ratio of 2 for scotopic vision illuminance using the spectral sensitivity $V^{\prime}(\lambda)$ of rods and photopic vision illuminance us ${ }^{-}$ ing standard relative luminous efficacy $V(\lambda)$, scotopic vision illuminance is 2 when photopic vision illuminance is 1 . When the input of the formula to calculate pupil diameter $D$ is converted from illuminance $E$ to scotopic vision illuminance $E_{S}$ based on the experimental conditions of Stanley et al. ${ }^{37)}$, Formula 10 is transformed into Formula 11.

$$
\begin{aligned}
& D=7.75-5.75\left\{\frac{\left(4135 \cdot E_{s} / 1692\right)^{0.41}}{\left(4135 \cdot E_{s} / 1692\right)^{0.41}+2}\right\} \\
& E_{s}=K_{s} \int_{380}^{780} P_{t}(\lambda) \cdot V^{\prime}(\lambda) d \lambda \\
& K_{s}=1700
\end{aligned}
$$

$D \quad$ : Pupil diameter (mm)

$E_{s} \quad$ : Scotopic vision illuminance (scotopic lx)

$K_{S} \quad$ : Maximum luminous efficacy of scotopic vision

$P_{t}(\lambda)$ : Spectral power distribution of light source $\left(\mathrm{W} / \mathrm{m}^{2}\right)$

$V^{\prime}(\lambda)$ : Standard relative luminous efficacy function of scotopic vision

2.5 Mathematical model for cases with pupil reflex Mathematical models for cases with pupil reflex focus 
on the quantity of light reaching the retina and are assumed to be able to be expressed by the product of pupil area and the amount corresponding to the illuminance using $M(\lambda)$. In the experiment by Brainard et al. ${ }^{22}$, the pupils of 72 subjects were dilated using medicine and the average pupil diameter was $7.19 \mathrm{~mm}$. For mathematical models for melatonin suppression values in cases with pupil reflex, the entry for Formula 6 for the amount corresponding to illuminance using $M(\lambda)$ was corrected with the product of the pupil area and the amount corresponding to illuminance using $M(\lambda)$, and is expressed by Formula 12.

$$
M S \% \text { normal }=\frac{0-66.9}{1+\left\{\left(E_{m} \cdot A\right) /\left(131 \cdot A_{f i x}\right)\right\}^{1.27}}+66.9
$$

$M S \%$ normal : Melatonin suppression value in cases with pupil reflex

$E_{m}$ : The amount corresponding to the illuminance using $M(\lambda)$

$A$ : Pupil area $\left(\mathrm{mm}^{2}\right)$

$A_{\text {fix }}$ : The pupil area in the experiment by Brainard et al.22) $\left(\mathrm{mm}^{2}\right)$

3. Verification of mathematical models

3.1 Existing reports used in the verification and verification results

The mathematical models to predict the melatonin suppression value in cases with pupil reflex and the actual experimental results were compared. For the actual experimental results in cases with pupil reflex, the results of McIntyre et al. ${ }^{30)}$, who used fluorescent lamps that simulate natural light, and Aoki et al. ${ }^{31}$, who used white fluorescent lamps, were used. Table 2 shows the details of the conditions and results for each experiment. As with the experiment by Brainard et al. which forms the basis of the mathematical models to predict the melatonin suppression value in this report, the experimental results used were the melatonin suppression value for 90 minutes of light exposure after control-adjustment.

Figure 2 shows the experimental results of McIntyre et al. ${ }^{30)}$ and the prediction results from the mathemati-

Table 2 Experimental condition and results of light-induced melatonin suppression for normal pupil ${ }^{3031)}$

\begin{tabular}{|c|c|c|c|c|c|c|}
\hline Author & $\begin{array}{l}\text { Number of } \\
\text { subjects }\end{array}$ & $\begin{array}{c}\text { Age (mean } \pm \\
\text { SD) }\end{array}$ & $\begin{array}{l}\text { Light exposure } \\
\text { time period }\end{array}$ & Light source & $\begin{array}{l}\text { Illuminance } \\
\text { (Ix) }\end{array}$ & $\begin{array}{l}\text { Mean percent } \\
\text { melatonin } \\
\text { suppression (\%) }\end{array}$ \\
\hline \multirow{3}{*}{ McIntyre et al. ${ }^{30)}$} & \multirow{3}{*}{6} & \multirow{3}{*}{$32.3 \pm 6.2$} & \multirow{3}{*}{$0: 00-1: 30$} & \multirow{3}{*}{ Vita-Lite } & 200 & 20 \\
\hline & & & & & 400 & 44 \\
\hline & & & & & 600 & 56 \\
\hline \multirow{4}{*}{ Aoki et al. ${ }^{311}$} & \multirow{4}{*}{5} & \multirow{4}{*}{$33.6 \pm 6.6$} & \multirow{4}{*}{$2: 00-3: 30$} & \multirow{4}{*}{$\begin{array}{c}\text { Cool-white } \\
\text { fluorescent } \\
\text { lamp }\end{array}$} & 500 & 22 \\
\hline & & & & & 1000 & 50 \\
\hline & & & & & 2500 & 63 \\
\hline & & & & & 5000 & 66 \\
\hline
\end{tabular}

cal model of this report. Figure 3 shows the experimental results of Aoki et al.31) and the prediction results from the mathematical model of this report. By considering the attenuation of the quantity of light reaching the retina due to pupil reflex, a lower melatonin suppression value was predicted in the high illuminance region, and it was possible to predict a value closer to actual experimental results compared to cases without pupil reflex. Comparisons with the experimental results showed that it was possible to roughly predict the maximum value of the melatonin suppression value. When the melatonin suppression value was in the midrange or lower, the predicted valued tended to be larger than the actual value.

\subsection{Discussion}

With regard to the disagreement between actual experimental results ${ }^{30) 31 \text { ) }}$ and mathematical models, this discussion will focus on the slope of the curve between the light intensity and the melatonin suppression value.

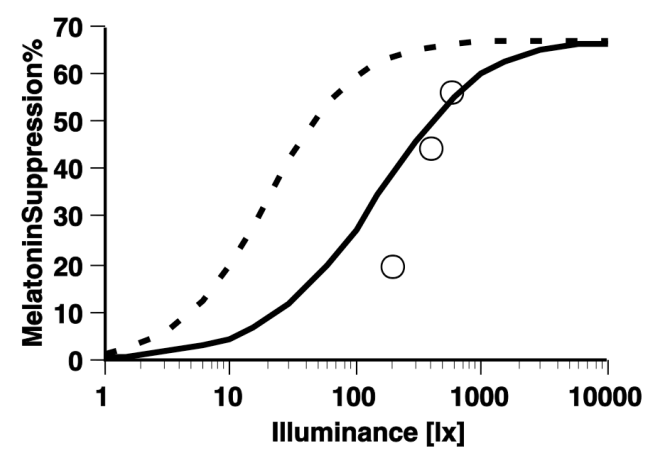

Figure 2 The open circle represents melatonin suppression \% from Mclntyre et $\mathrm{al}^{30)}$. The broken curve represents predicted melatonin suppression $\%$ for dilated pupil. The solid curve represents predicted melatonin suppression $\%$ for normal pupil.

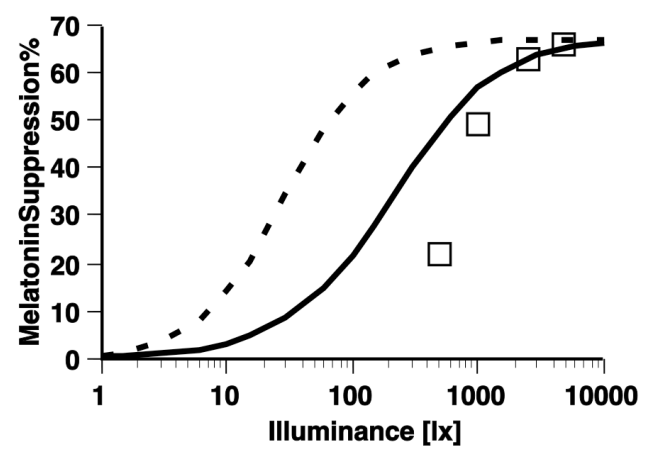

Figure 3 The open circle represents melatonin suppression \% from Aoki et $\mathrm{al}^{31}{ }^{1}$. The broken curve represents predicted melatonin suppression \% for dilated pupil. The solid curve represents predicted melatonin suppression $\%$ for normal pupil. 
When the slope of the curve is calculated $p$ of the logistic function of Formula 2, the results were as follows: McIntyre et al. ${ }^{30)}: 2.10$, Aoki et al. ${ }^{31)}: 2.52$, Brainard et al. ${ }^{22)}$ mathematical model without pupil reflex: 1.27 and the mathematical model for pupil reflex: 1.04. When broadband light was used in cases with pupil reflex, $p$ tended to be 2 or greater. When considering pupil reflex when monochromatic light was used in experiments without pupil reflex, it can be seen that $p$ lowers from a value slightly over 1 .

These results show that it is necessary to investigate an element other than those studied up until now in order to create mathematical models for melatonin suppression. This element is considered to be the action that causes steeper slope of curves for broadband light exposure compared to monochromatic light exposure and causes melatonin suppression values to further decrease when the prediction results for melatonin suppression values for monochromatic light exposure is in the mid to lower range.

There have been experiments that have used monochromatic and broadband lights to investigate melatonin suppression values in environments with pupil reflex ${ }^{28) 29)}$. Figueiro et al. used blue light emitting diodes (LED) and mercury lamps to test melatonin suppression $^{28)}$. When using an amount corresponding to the illuminance using an action spectrum model ${ }^{27)}$ for melatonin suppression and setting conditions such that the melatonin suppression value for mercury lamps was higher than that of blue light emitting diodes, the experimental results showed that the melatonin suppression value of mercury lamps was lower than that of blue light emitting diodes. Figueiro et al. also used mercury lamps and filtered mercury lamps with a wavelength of $436 \mathrm{~nm}$ to test melatonin suppression ${ }^{29)}$. When the product of the spectral radiation quantity and pupil area for the mercury lamps and $436 \mathrm{~nm}$ light was the same, the melatonin suppression value for mercury lamps was lower than that of the $436 \mathrm{~nm}$ light. With regard to these results, Figueiro et al. suggested the existence of an action spectrum with minus sensitivity $^{28)}$. However, assuming that Formula 12 represents the results of melatonin suppression experiments by Figueiro et al. using monochromatic light, results showing that melatonin suppression results from broadband light are lower than predicted do not contradict the fact that the experimental results of McIntyre et al. ${ }^{30)}$ and Aoki et al. ${ }^{31)}$ using broadband light were lower than the values predicted by Formula 12 in this report.

This suggests that, even when the product of the amount corresponding to illuminance using the action spectrum of melatonin suppression and the pupil area is the same, there is a mechanism at work that attenuates the melatonin suppression value for broadband light exposure compared to monochromatic light exposure. The spectral distributions of monochromatic and broadband light vary greatly. Monochromatic light has a narrow spectral radiation bandwidth while broadband light has a wide bandwidth. As a result, broadband light stimulates more photoreceptors in the retina simultaneously than monochromatic light. This suggests that there is a mechanism at work that attenuates melatonin suppression values when multiple photoreceptors are simultaneously stimulated.

\subsection{The introduction of attenuation items}

With broadband light exposure, it is assumed that there are items attenuating the melatonin suppression value compared to the mathematical model of Formula 12. The degree of attenuation is quantified as an attenuation coefficient (experimental value/predicted value) (Table 3). The attenuation coefficient is about 1 at high illuminance and lowers as illuminance drops.

Visual pigment in the retina is considered related to these attenuation items. The spectral sensitivity in the corneal positions is calculated from the spectral absorption $^{40)}$ of the crystalline lens and a template ${ }^{41)}$ indicating the spectral absorption characteristics of the visual pigment. The operating visual pigment as input of attenuation items was investigated in the visual pigment peak wavelength range of 400 to $600 \mathrm{~nm}$. With regard to the height of the relationship of each type of spectral sensitivity and attenuation coefficient, the relation between the amount corresponding to the illuminance using each type of spectral sensitivity and attenuation coefficient has been represented by the logistic function of Formula 2. From this result, it was learned that visual pigment with a peak wavelength at $469 \mathrm{~nm}$ had the highest relationship with the attenuation coefficient $\left(\mathrm{R}^{2}\right.$ $=0.77$ ). When the relationship between the photoreceptors in the retina of humans ${ }^{42)}$ and the attenuation coefficient is investigated, the coefficients of determination $\mathrm{R}^{2}$, which indicate the height of the relationship between the attenuation coefficient and the amount corresponding to the illuminance using the spectral sensitivity of $\mathrm{S}$ cones $\left(\lambda_{\max }=419 \mathrm{~nm}\right), \mathrm{M}$ cones $(530.8 \mathrm{~nm}), \mathrm{L}$ cones $(558.4 \mathrm{~nm})$, rods $(496 \mathrm{~nm})$, and ipRGC $\left(484 \mathrm{~nm}^{24)}\right)$

Table 3 Experimental results ${ }^{3031)}$ and predicted results, attenuation coefficient

\begin{tabular}{|c|c|c|c|c|c|}
\hline \multirow{2}{*}{ Author } & \multirow{2}{*}{ Light source } & \multirow{2}{*}{$\begin{array}{l}\text { Illuminance } \\
\text { (Ix) }\end{array}$} & \multicolumn{2}{|c|}{$\begin{array}{l}\text { Mean percent melatonin } \\
\text { suppression (\%) }\end{array}$} & \multirow{2}{*}{$\begin{array}{l}\text { Attenuation coefficien } \\
\text { (Experimental value / } \\
\text { Predicted value) }\end{array}$} \\
\hline & & & $\begin{array}{c}\text { Experimental } \\
\text { value }\end{array}$ & $\begin{array}{c}\text { Predicted } \\
\text { value }\end{array}$ & \\
\hline \multirow{3}{*}{ McIntyre et al. ${ }^{30)}$} & \multirow{3}{*}{ Vita-Lite } & 200 & 20 & 39 & 0.51 \\
\hline & & 400 & 44 & 50 & 0.88 \\
\hline & & 600 & 56 & 55 & 1.02 \\
\hline \multirow{4}{*}{ Aoki et al. ${ }^{31)}$} & \multirow{4}{*}{$\begin{array}{l}\text { Cool-white } \\
\text { florescent lamp }\end{array}$} & 500 & 22 & 48 & 0.46 \\
\hline & & 1000 & 50 & 57 & 0.88 \\
\hline & & 2500 & 63 & 63 & 1.00 \\
\hline & & 5000 & 66 & 65 & 1.02 \\
\hline
\end{tabular}




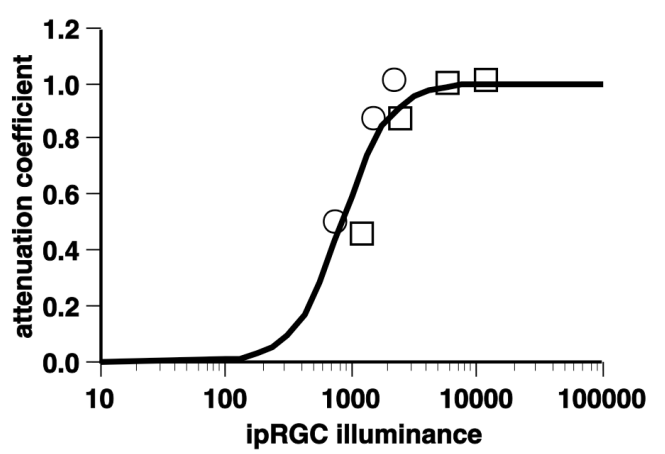

Figure 4 The bold curve represents relationship between illuminance using ipRGC spectral sensitivity and attenuation coefficient. The open circle represents attenuation coefficient of McIntyre's data. The open square represents attenuation coefficient of Aoki's data.

were $0.71,0.56,0.51,0.71$, and 0.75 respectively, which showed that ipRGC had the highest relationship with the attenuation coefficient.

Figure 4 shows the relationship between the amount corresponding to the illuminance using the spectral sensitivity of ipRGC and the attenuation coefficient. Because the amount corresponding to the illuminance using the spectral sensitivity of ipRGC is not defined, the calculation formula is expressed in Formula 13.

$$
\begin{aligned}
E_{i p R G C} & =K_{i p R G C} \int_{380}^{780} P_{t}(\lambda) \cdot i p R G C(\lambda) d \lambda \\
K_{i p R G C} & =3330
\end{aligned}
$$

$E_{i p R G C}$ : The amount corresponding to the illuminance using the spectral sensitivity of ipRGC

$K_{i p R G C}$ : Coefficient to set the value of $555 \mathrm{~nm}$ for ipR $G C(\lambda)$ to 683

$P_{t}(\lambda)$ : Spectral power distribution of light source $\left(\mathrm{W} / \mathrm{m}^{2}\right)$

ipRGC $(\lambda)$ : The spectral sensitivity of ipRGC in corneal positions

The attenuation coefficient is expressed as Formula 14. The melatonin suppression value when broadband light is used can be calculated by the product of the attenuation coefficient $\alpha$ of the Formula 14 and the melatonin suppression value $M S \%$ normal when there is $\mathrm{pu}^{-}$ pil reflex of Formula 12. Figure 5 shows the results predicted for the melatonin suppression value from the experiment conditions of McIntyre et al. ${ }^{30)}$ Similarly, Figure 6 shows the results predicted for the melatonin suppression value from the experiment conditions of Aoki et al. ${ }^{31}$ )

$$
\alpha=\frac{0-1}{1+\left(E_{i p R G C} / 845\right)^{2.26}}+1
$$

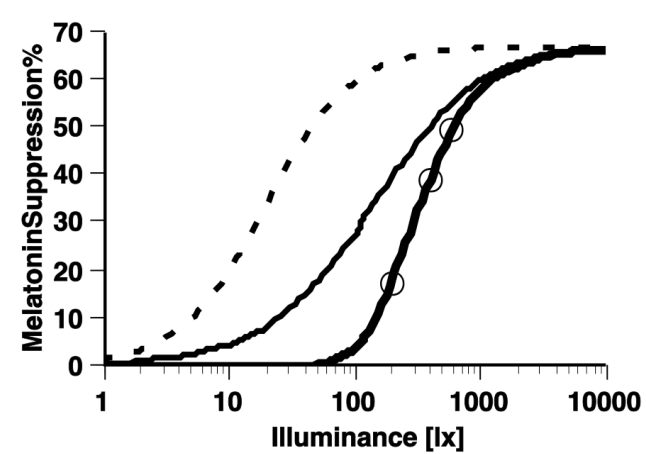

Figure 5 The open circle represents melatonin suppression \% from Mclntyre et $\mathrm{al}^{30}{ }^{30}$. The broken curve represents predicted melatonin suppression $\%$ for dilated pupil. The solid curve represents predicted melatonin suppression $\%$ for normal pupil. The bold curve represents predicted melatonin suppression \% for normal pupil contained attenuation factor.

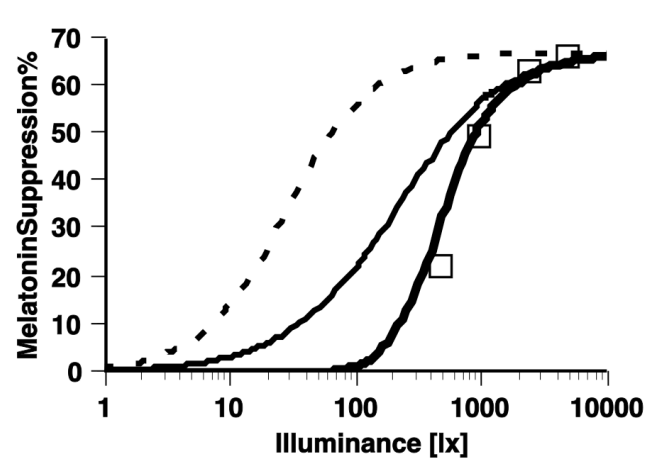

Figure 6 The open circle represents melatonin suppression \% from Aoki et $\mathrm{al}^{31)}$. The broken curve represents predicted melatonin suppression \% for dilated pupil. The solid curve represents predicted melatonin suppression $\%$ for normal pupil. The bold curve represents predicted melatonin suppression \% for normal pupil contained attenuation factor.

$\alpha \quad$ : Attenuation coefficient

$E_{i p R G C}$ : The amount corresponding to the illuminance using the spectral sensitivity of ipRGC

Figures 5 and 6 show that it is possible to almost completely predict the melatonin suppression value when broadband light is used by introducing attenuation items. Attenuation items have actions that cause the stimulus quantity of the ipRGC to attenuate the melatonin suppression value. This is analogous to $\mathrm{Neu}^{-}$ ropeptide $\mathrm{Y}$ of geniculohypothalamic tract $(\mathrm{GHT})^{46) 47)}$, one of the input routes to the $\mathrm{SCN}^{43)-45)}$ (Figure 7), which gives inhibitory input to the SCN. GHT outputs from the intergeniculate leaflet (IGL), a part of the lateral geniculate nucleus (LGN). The IGL is one of the neuron projections of the ipRGC25). This shows that the stimu- 


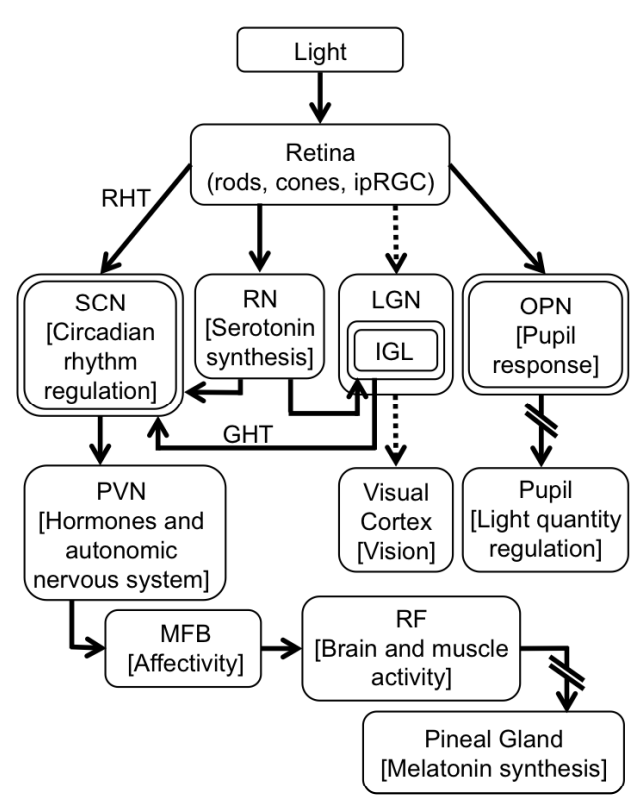

Figure 7 The solid line is non-image-forming pathway that reaches the pineal gland ${ }^{143)-45)}$. The dotted line is visual pathway that reaches the visual cortex. The ipRGC is projected to the regions enclosed in a double line ${ }^{25}$. There is an analogy between the attenuation factor and the activity of geniculohypothalamic tract $(\mathrm{GHT})^{46) 47)}$.

SCN: Suprachiasmatic nucleus, PVN: Paraventricular nucleus, MFB: Medial forebrain bundle, RF: Reticular formation, RN: Raphe nucleus, LGN: Lateral geniculate nucleus, IGL: Intergeniculate leaflet, OPN: Olivary pretectal nucleus, RHT: Retinohypothalamic tract, GHT: Geniculohypothalamic tract.

lus quantity of the ipRGC acting as an attenuation item input to attenuate the melatonin suppression value is analogous and consistent with the mechanism that input signals to the SCN .

\subsection{The action spectrum after the application of pupil reflex and attenuation items}

It is believed that the application of pupil reflex and attenuation items changes the action spectrum of melatonin suppression. Therefore, actions spectra were calculated using the various mathematical models created in this report. For the calculation, a gauss function was used to create monochromatic light with an FWHM of 1 $\mathrm{nm}$ and calculate the action spectrum for the range of 400 to $600 \mathrm{~nm}$ (Figure 8).

The mathematical model for cases with no pupil reflex based on the experimental results of Brainard et al. ${ }^{22)}$ (Formula 6) had a peak wavelength at $453 \mathrm{~nm}$ and results were similar to the experimental results of Brainard et al. ${ }^{22}$. The mathematical model considering changes of quantity of light reaching the retina in cases with pupil reflex based on the experimental results of Brainard et al. ${ }^{22}$ ) had a peak wavelength of $400 \mathrm{~nm}$. Be-

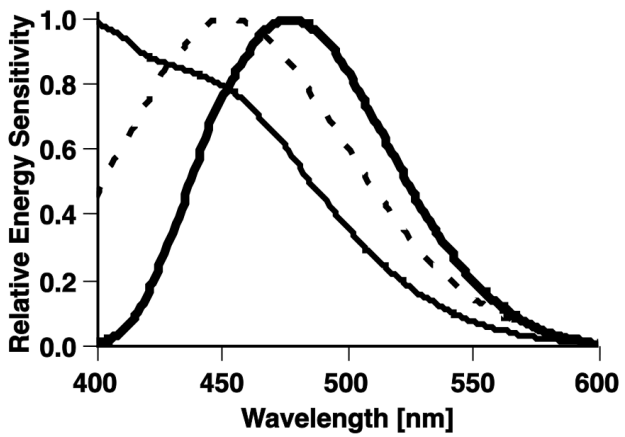

Figure 8 The broken curve represents action spectrum for melatonin suppression based on dilated pupil ${ }^{22}$. The solid curve represents action spectrum for melatonin suppression based on normal pupil. The bold curve represents action spectrum for melatonin suppression based on normal pupil, attenuation factor.

cause the short wavelength end was calculated only to $400 \mathrm{~nm}$ in this analysis, there in fact may be peak wavelengths for shorter wavelengths. This may be because since pupil reflex is considered, if the energy is large around the $507 \mathrm{~nm}$ peak wavelength of the rods, the pupil becomes smaller and because the quantity of light reaching the retina is attenuated, the peak wavelength may be away from the wavelength of $507 \mathrm{~nm}$. The mathematical model (the product of Formulas 12 and 14) considering the results of pupil reflex and attenuation items in the experimental results of Brainard et al. ${ }^{22)}$ had a peak wavelength of $478 \mathrm{~nm}$. This may be because since the peak wavelength for the spectral sensitivity, calculated from the template ${ }^{41)}$ expressing the spectral absorption of the lens and the spectral absorption characteristics of the visual pigment, is around 490 $\mathrm{nm}$ at the cornea position of the ipRGC, the attenuation coefficient moves toward 1 when there is a large amount of energy in this area and because this value moves toward 0 when moving away from the $490 \mathrm{~nm}$ area, the peak wavelength of the action spectrum comes to the peak wavelength area of the ipRGC. This analysis shows that when experimental results acquired using monochromatic light are applied to actual environments, the peak wavelength of the action spectrum changes greatly from the initial considered item.

\section{Review}

When creating mathematical models to predict melatonin suppression values under environments with pupil reflex, it was learned that it is not possible to sufficiently estimate actual experimental results ${ }^{30) 31)}$ simply by considering the effects ${ }^{37)}$ of changes in the quantity of light reaching the retina due to pupil reflex in the findings of melatonin suppression experiments ${ }^{22}$ using monochromatic light. This discrepancy in prediction has also been reported by Figueiro et al.28)29) who suggested 
there is a mechanism operating that attenuates the melatonin suppression value when the light source is broadband light versus monochromatic light. These findings are considered important discoveries that suggest the direction of future research when attempting to cultivate a better understanding of the non-image forming effects of light.

The mechanism that attenuates the melatonin suppression value during broadband light exposure compared to monochromatic light exposure has been called an attenuation item. Attenuation items are a multiplier for the attenuation coefficient in the prediction results for melatonin suppression values with monochromatic light. Presuming visual pigment is working as attenuation item input suggests that there exist photoreceptors with a peak wavelength at $469 \mathrm{~nm}$. However, since such photoreceptors do not exist, it is believed that multiple photoreceptors input attenuation items or that there is some other mechanism involved. Out of the photoreceptors that have been confirmed to exist, it has been suggested that the ipRGC are most likely to act as an input of attenuation items. The action of attenuation items is analogous to NPY46)47) of the GHT, one of the input routes to the SCN, which give inhibitory input to the SCN. GHT is output from the IGL, which is believed to be comparable to the Koniocellular layer (K layer) of the LGN. It has been confirmed that the ipRGC project to the IGL ${ }^{25}$ 48). Furthermore, since the K layer processes blue-yellow contrast color information, it is believed that the IGL also performs signal processing for these cones. The above shows that the action of attenuation items is similar to the actions of NPY of the GHT, one of the input routes to the $\mathrm{SCN}$, and is analogous to a physiological mechanism. Furthermore, projections from ipRGC have been confirmed in the IGL, which is the output source of the GHT. Since it has been suggested that various types of cones are also inputting, it is been suggested that it is possible that the attenuation item input signals are processed here. In this report, it has been assumed that attenuation items do not function during exposure to monochromatic light but function during exposure to broadband light. However, further research is necessary regarding whether mechanisms like the attenuation items assumed in this report actually exist, and if they exist, what actions do they have under different lighting conditions.

It is been indicated that the introduction of pupil reflex and attenuation items change the action spectrum of melatonin suppression. Therefore, it is been suggested that the pupil reflex and attenuation change not only the quantity of light reaching the retina and the attenuation of the melatonin secretion value, but also the action spectrum. While this report indicates that there are cases in which the action spectrum of mela- tonin suppression value changes, further research is required regarding whether such changes are actually occurring, and if they are occurring, under which conditions do they occur. The idea that attenuation items do not act with monochromatic light and act with broadband light in order to explain the findings of this report and the reports of Figueiro et al. ${ }^{28) 29)}$ may be useful when attempting to clarify this mechanism.

With regard to the various non-image forming effects of light, it has been suggested that light signals are input to the SCN and the various parts of the route that transfer it to the pineal gland are related. Therefore, it may be possible to use the findings from the analysis of the melatonin suppression effects presented here attempting to understand effects on other marks. Findings regarding the non-image forming effects of light ${ }^{2)-16}$ ) on marks other than melatonin such as brain waves and electrocardiograms have shown different effects by color depending on the type of light source. LED light sources have received attention because of their low power usage and long life. If their use becomes widespread, it is expected that it will become more difficult to indicate the effects on the body by color name because its spectral distribution differs from other sources such as incandescent lamps, fluorescent lamps, and HID lamps. Therefore, it is considered important to investigate the non-image forming effects of light on bodily functions using dedicated markers.

In a press release dated December 2007, the International Agency for Research on Cancer announced that it intended to classify shift work that involves circadian disruption as a Group 2A carcinogen (meaning it is probably carcinogenic to humans $)^{49}$. The report stated that people who perform work that disrupts circadian rhythms are at high risk for breast cancer ${ }^{50)}$-52) or prostate cancer53). The IARC report is believed to be the first by an official organization that acknowledged the relationship between circadian rhythms and cancer. It has been reported that melatonin suppresses the growth of cancer ${ }^{54)}$ and it has been strongly suggested that the suppression of melatonin secretion has an important effect on cancer production. Therefore, clarifying the effects of light on melatonin secretion will provide important findings for the investigation of the effects of light on cancer production.

The report by Stanley et al. ${ }^{37)}$ was used when estimating pupil reflex by light, and the accommodation of $\mathrm{pu}^{-}$ pils by the spectral sensitivity of rods was used ${ }^{39}$. However, it has been reported that the ipRGC is involved in pupil reflex because the ipRGC projects to the OPN within the brain, which adjusts pupil reflex ${ }^{25}$. It is also been reported from experiments involving humans that spectral sensitivity of the ipRGC was seen by pupil reflex ${ }^{55) 56)}$. The light intensity level at which the ipRGC 
reacts is higher than both rods and cones ${ }^{26) 48)}$, and it has been suggested that ipRGC adjusts pupil reflex around the minimum diameter ${ }^{26) 55)}$. Therefore, it is necessary to consider the effects of ipRGC when creating predictive formulas for pupil diameter. Using the abovementioned consideration, it may be possible to decrease the difference between the spectral sensitivity of visual pigment assumed as an input of attenuation items and the spectral sensitivity of actual visual pigment.

\section{Summary}

Mathematical models were created to predict the melatonin suppression effects from light exposure at night, which is one of the non-image forming effects of light. When considering the effects of pupil reflex ${ }^{37)}$ in the findings of melatonin suppression experiments ${ }^{22)}$ using monochromatic light, it was not possible to sufficiently

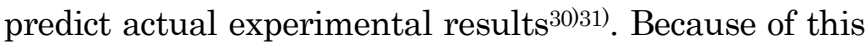
discrepancy, and in order to predict the melatonin suppression effects of both monochromatic and broadband light from the existing research of Figueiro et al.28)29), it was necessary to assume a mechanism that has not been hypothesized up until now. The existence of an attenuation mechanism was assumed. The action of attenuation items was analogous to the physiological mechanism around the SCN. Furthermore, it was indicated that pupil reflex and attenuation items change the action spectrum. Based on the above, it may be possible to explain this mechanism in detail by analyzing experimental conditions under which the action spectrum changed.

\section{Acknowledgments}

This report was completed with the assistance of the 15th Illuminating Engineering Institute of Japan (2006) Study and Educational Grants plans (research related to biological responses involving ipRGC). We are thankful to the members of the Research and Investigation Committee of the Illuminating Engineering Institute of Japan (the research studies committee investigating lighting environment design principles with regard to physiological effects), Illumination Research Section of the Japan Society of Physiological Anthropology, and the Chiba Vision Meeting for their valuable comments when summarizing this research.

\section{Reference}

(1) Klein, D. C., Smoot, R., Weller, J. L., Higa, S., Markey, S. P., Creed, G. J. and Jacobowitz, D. M. : Lesions of the paraventricular nucleus area of the hypothalamus disrupt the suprachiasmatic leads to spinal cord circuit in the melatonin rhythm generat- ing system, Brain Res Bull, 10-5, pp.647-652 (1983).

(2) Ali, M. R.: Pattern of EEG recovery under photic stimulation by light of different colors, Electroencephalogr Clin Neurophysiol, 33-3, pp.332-335 (1972).

(3) Shimagami, K. and Hihara, M.: Effects of color lights on man in sense of environmental image and condition of mind and body, Japanese Journal of Hygiene, 446-1, p.135 (1991) (in Japanese).

(4) Shimagami, K. and Hihara, M.: Effects of color lights on man in sense of environmental image and condition of mind and body, 2nd Report: In a case of changing the room lighting to color lights, Japanese Journal of Hygiene, 47-1, p.148 (1992) (in Japanese).

(5) Deguchi, T. and Sato, M.: The effect of color temperature of lighting sources on mental activity level, Ann Physiol Anthrop, 11-1, pp.37-43 (1992).

(6) Sato, M.: A Study on Amenity of Indoor Illuminations. Including the Effects of Indoor Climate. Annual Report of Housing Research Foundation, 20, pp.305-316 (1993) (in Japanese).

(7) Küller, R. and Wetterberg, L.: Melatonin, cortisol, EEG, ECG and subjective comfort in healthy humans : Impact of two fluorescent lamp types at two light intensities, Lighting Res Technol, 25-2, pp.7181 (1993).

(8) Iwakiri, K., Watanuki, S., Yasukouchi, A. and Tochihara, Y: The Effect and After-effect of Lightsource Color on Early Component of CNV, Japanese Journal of Physiological Anthropology, 2-3, pp.139145 (1997) (in Japanese).

(9) Noguchi, H. and Sakaguchi, T: Effect of illuminance and color temperature on lowering of physiological activity, Appl Human Sci, 18-4, pp.117-123 (1999).

(10) Omori, M., Hashimoto, R. and Kato, Y.: Relation between Psychological and Physiological Responses on Color Stimulus, Journal of The Color Science Association of Japan, 26-2, pp.50-63 (2002) (in Japanese).

(11) Ueda, Y., Hayashi, K., Kuroiwa, K., Miyoshi, N., Kashiba, H. and Takeda, D.: Consciousness and recognition of five colors - Using Functional-MRI and brain wave measurments - , J Intl Soc Life Info Sci, 22-2, pp.366-371 (2004).

(12) Katsuura, T., Yasuda, T., Shimomura, Y. and Iwanaga, K.: Effects of monochromatic light on time sense for short intervals, J Physiol Anthropol, 26-2, pp.95-100 (2007).

(13) Lee, H., Katsuura, T., Iwanaga, K., Shimomura, Y., Higashi, H. and Ichijo, T.: The Effect of Monochromatic Light Exposure on Human Physiological Responses, Japanese Journal of Physiological Anthropology, 13-2, pp.75-78 (2008) (in Japanese).

(14) Jacobs, K. W. and Hustmyer Jr, F. E.: Effects of four 
psychological primary colors on GSR, heart rate and respiration rate, Percept Mot Skills, 38-3, pp.763766 (1974).

(15) Kobayashi, H. and Sato, M.: Physiological responses to illuminance and color temperature of lighting, Ann Physiol Anthrop, 11-1, pp.45-49 (1992).

(16) Schäfer, A. and Kratky, K. W.: The effect of colored illumination on heart rate variability, Forsch Komplementmed, 13-3, pp.167-173 (2006).

(17)Lewy, A. J., Wehr, T. A., Goodwin, F. K., Newsome, D. A. and Markey, S. P.: Light suppresses melatonin secretion in humans, Science, 210-4475, pp.12671269 (1980).

(18) Hollwich, F. and Dieckhues, B.: The effect of natural and artificial light via the eye on the hormonal and metabolic balance of animal and man, Ophthalmologica, 180-4, pp.188-197 (1980).

(19) Cauter, E. Van, Sturis, J., Byrne, M. M., Blackman, J. D., Leproult, R., Ofek, G., L'Hermite-Balériaux, M., Refetoff, S., Turek, F. W. and Reeth, O. Van: Demonstration of rapid light-induced advances and delays of the human circadian clock using hormonal phase markers, Am J Physiol, 266-6, pp.E953-E963 (1994).

(20) Morita, T., Teramoto, Y. and Tokura, H.: Inhibitory effect of light of different wavelengths on the fall of core temperature during the nighttime, Jpn J Physiol, 45-4, pp.667-671 (1995).

(21) Morita, T. and Tokura, H.: Effects of lights of different color temperature on the nocturnal changes in core temperature and melatonin in humans, Appl Human Sci, 15-5, pp.243-246 (1996).

(22)Brainard, G. C., Hanifin, J. P., Greeson, J. M., Byrne, B., Glickman, G., Gerner, E. and Rollag, M. D.: Action spectrum for melatonin regulation in humans: evidence for a novel circadian photoreceptor, J Neurosci, 21-16, pp.6405-6412 (2001).

(23) Thapan, K., Arendt, J. and Skene, D. J.: An action spectrum for melatonin suppression: evidence for a novel non-rod, non-cone photoreceptor system in humans, J Physiol, 535-1, pp.261-267 (2001).

(24) Berson, D. M., Dunn, F. A. and Takao, M.: Phototransduction by retinal ganglion cells that set the circadian clock, Science, 295-5557, pp.1070-1073 (2002).

(25) Hattar, S., Liao, H. W., Takao, M., Berson, D. M. and Yau, K. W.: Melanopsin-containing retinal ganglion cells: architecture, projections, and intrinsic photosensitivity, Science, 295-5557, pp.1065-1070 (2002).

(26) Panda, S., Provencio, I., Tu, D. C., Pires, S. S., Rollag, M. D., Castrucci, A. M., Pletcher, M. T., Sato, T. K., Wiltshire, T., Andahazy, M., Kay, S. A., Van Gelder R. N. and Hogenesch, J. B.: Melanopsin is required for non-image-forming photic responses in blind mice, Science, 301-5632, pp.525-527 (2003).

(27) Rea, M. S., Bullough, J. D. and Figueiro, M. G.: Phototransduction for human melatonin suppression, J Pi-neal Res, 32-4, pp.209-213 (2002).

(28) Figueiro, M. G., Bullough, J. D., Parsons, R. H. and Rea, M. S.: Preliminary evidence for spectral opponency in the suppression of melatonin by light in humans, Neuroreport, 15-2, pp.313-316 (2004).

(29) Figueiro, M. G., Bullough, J. D., Bierman, A. and Rea, M. S.: Demonstration of additivity failure in human circadian phototransduction, Neuro Endocrinol Lett, 26-5, pp.493-498 (2005).

(30) McIntyre, I. M., Norman, T. R., Burrows, G. D. and Armstrong, S. M: Quantal melatonin suppression by exposure to low intensity light in man, Life Sci, 45-4, pp.327-332 (1989).

(31) Aoki, H., Yamada, N., Ozeki, Y., Yamane, H. and Kato, N.: Minimum light intensity required to suppress nocturnal melatonin concentration in human saliva, Neurosci Lett, 252-2, pp.91-94 (1998).

(32) Gaddy, J. R., Rollag, M. D. and Brainard, G. C.: Pupil size regulation of threshold of light-induced melatonin suppression, J Clin Endocrinol Metab, 77-5, pp.1398-1401 (1993).

(33) Herljevic, M., Middleton, B., Thapan, K. and Skene, D. J.: Light-induced melatonin suppression: agerelated reduction in response to short wavelength light, Exp Gerontol, 40-3, pp.237-242 (2005).

(34) McIntyre, I. M., Norman, T. R., Burrows, G. D. and Armstrong, S. M.: Human melatonin response to light at different times of the night, Psychoneuroendocrinology, 14-3, pp.187-193 (1989).

(35) Glickman, G., Levin, R. and Brainard, G. C.: Ocular input for human melatonin regulation: relevance to breast cancer, Neuro Endocrinol Lett, 23-Suppl 2, pp.17-22 (2002).

(36) Partridge, J. C. and De Grip, W. J.: A new template for rhodopsin (vitamin A1 based) visual pigments, Vision Res, 31-4, pp.619-630 (1991).

(37) Stanley, P. A. and Davies, A. K. The effect of field of view size on steady-state pupil diameter, Ophthal Physiol Opt, 15-6, pp.601-603 (1995).

(38) Ohta, N.: Color Engineering, Tokyo Denki University Press, pp.16-21 (1993) (in Japanese).

(39) Wagman, I. H. and Gullberg, J. E.: The relationship between monochromatic light and pupil diameter. The low intensity visibility curve as measured by pupillary measurements, Am J Physiol, 137-4, pp.769-778 (1942).

(40) Stockman, A., Sharpe, L. T. and Fach, C.: The spectral sensitivity of the human short-wavelength sensitive cones derived from thresholds and color matches, Vision Res, 39-17, pp.2901-2927 (1999).

(41)Lamb, T. D.: Photoreceptor spectral sensitivities: 
common shape in the long-wavelength region, $\mathrm{Vi}^{-}$ sion Res, 35-22, pp.3083-3091 (1995).

(42) Dartnall, H. J., Bowmaker, J. K. and Mollon, J. D.: Human visual pigments: microspectrophotometric results from the eyes of seven persons, Proc R Soc Lond B BiolSci, 220-1218, pp.115-130 (1983).

(43) Cagampang, F. R., Yamazaki, S., Otori, Y. and Inouye, S. I.: Serotonin in the raphe nuclei: regulation by light and an endogenous pacemaker, Neuroreport, 5-1, pp.49-52 (1993).

(44) Morin, L. P.: Serotonin and the regulation of mammalian circadian rhythmicity, Ann Med, 31-1, pp.1233 (1999).

(45) Arita, H.: Serotonin Nervous System, update [3] Effect of biological clock on entrainment mechanism, Clinical Neuroscience, 21-6, pp.616-617 (2003) (in Japanese).

(46) Albers, H. E. and Ferris, C. F.: Neuropeptide Y: role in light-dark cycle entrainment of hamster circadian rhythms, Neurosci Lett, 50-1-3, pp.163-168 (1984).

(47) Weber, E. T. and Rea, M. A.: Neuropeptide Y blocks light-induced phase advances but not delays of the circadian activity rhythm in hamsters, Neurosci Lett, 231-3, pp.159-162 (1997).

(48) Dacey, D. M., Liao, H. W., Peterson, B. B., Robinson, F. R., Smith, V. C., Pokorny, J., Yau, K. W. and Gamlin, P. D.: Melanopsin-expressing ganglion cells in primate retina signal colour and irradiance and project to the LGN, Nature, 433-7027, pp.749-754 (2005).

(49) Straif, K., Baan, R., Grosse, Y., Secretan, B., Ghissassi, F. El., Bouvard, V., Altieri, A., BenbrahimTallaa, L. and Cogliano, V.: Carcinogenicity of shiftwork, painting, and fire-fighting, Lancet Oncol, 8-12, pp.1065-1066 (2007).

(50) Pukkala, E., Auvinen, A. and Wahlberg, G.: Inci- dence of cancer among Finnish airline cabin attendants, 1967-92, BMJ, 311-7006, pp.649-652 (1995).

(51) Tynes, T., Hannevik, M., Andersen, A., Vistnes, A. I. and Haldorsen, T: Incidence of breast cancer in Norwegian female radio and telegraph operators, Cancer Causes Control, 7-2, pp.194-204 (1996).

(52) Hansen, J.: Increased breast cancer risk among women who work predominantly at night, Epidemiology, 12-1, pp.74-77 (2001).

(53) Kubo, T., Ozasa, K., Mikami, K., Wakai, K., Fujino, Y., Watanabe, Y., Miki, T., Nakao, M., Hayashi, K., Suzuki, K., Mori, M., Washio, M., Sakauchi, F., Ito, Y., Yoshimura, T. and Tamakoshi, A.: Prospective cohort study of the risk of prostate cancer among rotating-shift workers: findings from the Japan collaborative cohort study, Am J Epidemiol, 164-6, pp.549-555 (2006).

(54) Blask, D. E., Brainard, G. C., Dauchy, R. T., Hanifin, J. P., Davidson, L. K., Krause, J. A., Sauer, L. A., Rivera-Bermudez, M. A., Dubocovich, M. L., Jasser, S. A., Lynch, D. T., Rollag, M. D. and Zalatan, F.: Melatonin-depleted blood from premenopausal women exposed to light at night stimulates growth of human breast cancer xenografts in nude rats, Cancer Res, 65-23, pp.11174-11184 (2005).

(55) Takahashi, Y., Katsuura, T., Iwanaga, K. and Shimomura, Y.: Action Spectrum for Pupillary Light Reflex at High Irradiances, Proceedings of Lecture in 5th Young Wave Forum, The Illuminating Engineering Institute of Japan, pp.49-54 (2006) (in Japanese).

(56) Gamlin, P. D., McDougal, D. H., Pokorny, J., Smith, V. C., Yau, K. W. and Dacey, D. M.: Human and macaque pupil responses driven by melanopsincontaining retinal ganglion cells, Vision Res, 47-7, pp.946-954 (2007). 